PROCEEDINGS OF THE

AMERICAN MATHEMATICAL SOCIETY

Volume 137, Number 3, March 2009, Pages 1081-1091

S 0002-9939(08)09630-5

Article electronically published on October 3, 2008

\title{
UNIFORMLY CONVEX FUNCTIONS ON BANACH SPACES
}

\author{
J. BORWEIN, A. J. GUIRAO, P. HÁJEK, AND J. VANDERWERFF
}

(Communicated by N. Tomczak-Jaegermann)

\begin{abstract}
Given a Banach space $(X,\|\cdot\|)$, we study the connection between uniformly convex functions $f: X \rightarrow \mathbb{R}$ bounded above by $\|\cdot\|^{p}$ and the existence of norms on $X$ with moduli of convexity of power type. In particular, we show that there exists a uniformly convex function $f: X \rightarrow \mathbb{R}$ bounded above by $\|\cdot\|^{2}$ if and only if $X$ admits an equivalent norm with modulus of convexity of power type 2 .
\end{abstract}

\section{INTRODUCTION}

Uniformly convex functions on Banach spaces were introduced by Levitin and Poljak in 10. Their properties were studied in depth by Zălinescu [13, and then later Azé and Penot [1] studied their duality with uniformly smooth convex functions. The monograph [14] provides a systematic development of these topics. Additionally, related properties of convex functions and their applications have been studied in papers such as 2, 3, 4, 5. In particular, 3] examines various properties of $\|\cdot\|^{r}$ when $\|\cdot\|$ is a uniformly convex norm. In this note, we will present a related result that determines when functions of the form $f=\|\cdot\|^{r}$ are uniformly convex. We also examine a more general converse problem: if $f: X \rightarrow \mathbb{R}$ is uniformly convex and bounded above by $\|\cdot\|^{r}$, does $X$ admit a norm with a modulus of convexity of power type related to $r$ ?

We work with a real Banach space $(X,\|\cdot\|)$ with dual $X^{*}$, and let $B_{X}$ and $S_{X}$ denote the closed unit ball and unit sphere respectively. The modulus of convexity of a norm $\|\cdot\|$ on $X$ is defined for $\varepsilon \in[0,2]$ by

$$
\delta_{\|\cdot\|}(\varepsilon)=\inf \left\{1-\frac{1}{2}\|x+y\|: \quad x, y \in S_{X},\|x-y\| \geq \varepsilon\right\} .
$$

The norm $\|\cdot\|$ is called uniformly convex if $\delta_{\|\cdot\|}(\varepsilon)>0$ for all $\varepsilon \in(0,2]$; additionally, we say that $\|\cdot\|$ has modulus of convexity of power type $p$ if there exists $C>0$ so that $\delta_{\|\cdot\|}(\varepsilon) \geq C \varepsilon^{p}$ for $\varepsilon \in[0,2]$. Note that from [11] it follows that $p \geq 2$. The

Received by the editors March 16, 2007, and, in revised form, April 26, 2008.

2000 Mathematics Subject Classification. Primary 52A41, 46G05, 46N10, 49J50, $90 \mathrm{C} 25$.

Key words and phrases. Convex function, uniformly smooth, uniformly convex, superreflexive.

The first author's research was supported by NSERC and the Canada Research Chair Program.

The second author's research was supported by the grants MTM2005-08379 of MECD (Spain), 00690/PI/04 of Fundación Séneca (CARM, Spain) and AP2003-4453 of MECD (Spain).

The third author's research was supported by the grants A100190502, IAA 100190801 and Inst. Research Plan AV0Z10190503. 
modulus of smoothness of the norm $\|\cdot\|$ is defined for $\tau>0$ by

$$
\rho_{\|\cdot\|}(\tau)=\sup \left\{\frac{\|x+\tau y\|+\|x-\tau y\|}{2}-1: \quad\|x\|=\|y\|=1\right\} .
$$

The norm $\|\cdot\|$ is called uniformly smooth if $\lim _{\tau \rightarrow 0^{+}} \rho_{\|\cdot\|}(\tau) / \tau=0$; additionally, we say that $\|\cdot\|$ has modulus of smoothness of power type $q$ if there exists $C>0$ such that $\rho_{\|\cdot\|}(\tau) \leq C \tau^{q}$ for $\tau>0$. It follows also from [11] that $q \leq 2$. See [6, Chapter IV] for more information on these notions.

We now introduce the like-named concepts for convex functions whose definitions are different from - but motivated by - the norm cases. Given a convex function $f$ : $X \rightarrow(-\infty,+\infty]$ we define its modulus of convexity as the function $\delta_{f}:(0,+\infty) \rightarrow$ $[0,+\infty]$ given by

$$
\delta_{f}(t):=\inf \left\{\frac{1}{2} f(x)+\frac{1}{2} f(y)-f\left(\frac{x+y}{2}\right): \quad\|x-y\|=t, x, y \in \operatorname{dom} f\right\}, \quad t>0,
$$

where the infimum over the empty set is $+\infty$. We say that $f$ is uniformly convex when $\delta_{f}(t)>0$ for all $t>0$; additionally, we say that $f$ has a modulus of convexity of power type $p$ if there exists $C>0$ so that $\delta_{f}(t) \geq C t^{p}$ for all $t>0$.

Similarly we consider the modulus of smoothness of the convex function $f: X \rightarrow$ $(-\infty,+\infty)$ as the function $\rho_{f}:(0,+\infty) \rightarrow[0,+\infty]$ defined by

$$
\rho_{f}(t):=\sup \left\{\frac{1}{2} f(x)+\frac{1}{2} f(y)-f\left(\frac{x+y}{2}\right): \quad\|x-y\|=t\right\}, \quad t>0 .
$$

We will say $f$ is uniformly smooth if $\lim _{t \rightarrow 0^{+}} \rho_{f}(t) / t=0$; additionally we say that $f$ has a modulus of smoothness of power type $q$ if there is a constant $C>0$, so that $\rho_{f}(t) \leq C t^{q}$ for all $t>0$.

This terminology may cause some confusion, because, for example, $f=\|\cdot\|$ is never uniformly convex as a function, even when $\|\cdot\|$ is a uniformly convex norm. Therefore, it is important to note the context in which the terms are used. Moreover, the concepts of uniform smoothness and uniform convexity for functions are sometimes defined using the gage of uniform convexity and gage of uniform smoothness respectively as found in [14. It is important to note that these alternate definitions using the respective gages are equivalent to those just given; cf. 13, Remark 2.1] and [14, p. 205].

In contrast to the situation for norms, neither the restriction $p \geq 2$ for the modulus of convexity of a convex function nor $q \leq 2$ for the modulus of smoothness of a convex function are necessary. To see this, consider an indicator function of a single point and a constant function respectively.

It is also instructive to consider the function $f=|\cdot|^{r}$ on the real line where $r>1$ is fixed. For $1<r \leq 2$, one can check that $f^{\prime}$ satisfies an $(r-1)$-Hölder condition. Then using the Mean Value Theorem, it follows that $f$ has a modulus of smoothness of power type $r$. On the other hand, for $t>0$, when taking $x=\frac{t}{2}$, $y=-\frac{t}{2}$, we have $\rho_{f}(t) \geq \frac{1}{2}\left(\frac{t}{2}\right)^{r}+\frac{1}{2}\left(\frac{t}{2}\right)^{r}=\frac{1}{2^{r}} t^{r}$. Hence $f$ does not have a modulus of smoothness of power type $q$ where $q<r$. In the case $r>2$, it is straightforward to check that $f$ is not uniformly smooth. Dually, but certainly not trivially, when $r \geq 2, f$ is uniformly convex with modulus of convexity of power type $r$. The next section will elaborate on results of this nature in a more general setting. 
Finally, the Fenchel conjugate of $f: X \rightarrow(-\infty,+\infty]$ is the function $f^{*}: X^{*} \rightarrow$ $[-\infty,+\infty]$ defined by

$$
f^{*}\left(x^{*}\right)=\sup \left\{x^{*}(x)-f(x): \quad x \in X\right\}, \quad x^{*} \in X^{*} .
$$

It is through this concept that duality between uniform convexity and uniform smoothness is studied in the context of convex functions; see [1, 14].

\section{UNIFORM CONVEXITY OF FUNCTIONS AND NORMS}

This section will demonstrate for $2 \leq p<\infty$ that $f=\|\cdot\|^{p}$ is uniformly convex if and only if the norm $\|\cdot\|$ has modulus of convexity of power type $p$.

Lemma 2.1. Let $0<r \leq 1$. Then $\left|t^{r}-s^{r}\right| \leq|t-s|^{r}$ for all $s, t \in[0, \infty)$.

Proof. For $x \geq 0$ we have $(1+x)^{r} \leq 1+x^{r}$ (see [12, Example 4.20]). Setting $x=(t-s) / s$ with $t \geq s>0$, and then multiplying by $s^{r}$, we get $t^{r} \leq s^{r}+(t-s)^{r}$. The conclusion follows from this.

Theorem 2.2. For $1<q \leq 2$, the following are equivalent in a Banach space $(X,\|\cdot\|)$.

(a) The norm $\|\cdot\|$ has modulus of smoothness of power type $q$.

(b) The derivative of $f=\|\cdot\|^{q}$ exists everywhere and satisfies a $(q-1)$-Hölder condition.

(c) The function $f=\|\cdot\|^{q}$ has modulus of smoothness of power type $q$.

(d) The function $f=\|\cdot\|^{q}$ is uniformly smooth.

Proof. (a) $\Rightarrow$ (b): Assume that $\|\cdot\|$ has modulus of smoothness of power type $q$. According to [6, Lemma IV.5.1], the norm $\|\cdot\|$ has (Fréchet) derivative at each point $x \in X \backslash\{0\}$ - call it $\phi_{x}$-and moreover it satisfies on $S_{X}$ a Hölder condition; that is, there is $C>0$ so that

$$
\left\|\phi_{x}-\phi_{y}\right\| \leq C\|x-y\|^{q-1} \text { for all } x, y \in S_{X} .
$$

Let $f=\|\cdot\|^{q}$. Then $f^{\prime}(0)=0$, and $f^{\prime}(x)=q\|x\|^{q-1} \phi_{x}$ for $x \neq 0$. Thus if $x=0$ or $y=0$, then $\left\|f^{\prime}(x)-f^{\prime}(y)\right\| \leq q\|x-y\|^{q-1}$. Let $x, y \in X \backslash\{0\}$. Then

$$
\begin{aligned}
f^{\prime}(x)-f^{\prime}(y) & =q\|x\|^{q-1} \phi_{x}-q\|y\|^{q-1} \phi_{y} \\
& =q\|x\|^{q-1}\left(\phi_{x}-\phi_{y}\right)+\left(q\|x\|^{q-1}-q\|y\|^{q-1}\right) \phi_{y} .
\end{aligned}
$$

Using Lemma 2.1 we also compute

$$
\left|q\|x\|^{q-1}-q\|y\|^{q-1}\right| \leq q|\|x\|-\|y\||^{q-1} \leq q\|x-y\|^{q-1} .
$$

We now work on an estimate for $q\|x\|^{q-1}\left\|\phi_{x}-\phi_{y}\right\|$. We may and do assume that $0<\|y\| \leq\|x\|$. If $\|y\| \leq\|x\| / 2$, then

$$
q\|x\|^{q-1}\left\|\phi_{x}-\phi_{y}\right\| \leq 2 q\|x\|^{q-1} \leq q 2^{q}\|x-y\|^{q-1} .
$$

Further, assume that $\|y\| \geq\|x\| / 2$. Consider $x^{\prime}=\lambda x$ where $\lambda=\|y\| /\|x\|$, so that $\left\|x^{\prime}\right\|=\|y\|$. Then

$$
\left\|x^{\prime}-y\right\| \leq\left\|x^{\prime}-x\right\|+\|x-y\|=\|x\|-\|y\|+\|x-y\| \leq 2\|x-y\| .
$$


Now let $\alpha=\|y\|$. Observe that $\phi_{x}$ and $\phi_{y}$ are also support functionals for $\alpha^{-1} x^{\prime}$ and $\alpha^{-1} y$ respectively. Applying (2.1), the fact that $\|x\| \leq 2 \alpha$, and (2.5), we obtain

$$
\begin{aligned}
\left\|\phi_{x}-\phi_{y}\right\| & \leq C\left\|\alpha^{-1} x^{\prime}-\alpha^{-1} y\right\|^{q-1} \leq \frac{C}{\alpha^{q-1}}\left\|x^{\prime}-y\right\|^{q-1} \\
& \leq \frac{C 2^{q-1}}{\|x\|^{q-1}}(2\|x-y\|)^{q-1}=\frac{C 4^{q-1}}{\|x\|^{q-1}}\|x-y\|^{q-1} .
\end{aligned}
$$

Consequently, $q\|x\|^{q-1}\left\|\phi_{x}-\phi_{y}\right\| \leq C 4^{q-1} q\|x-y\|^{q-1}$. This inequality and (2.4) show there exists $K>0$ such that

$$
q\|x\|^{q-1}\left\|\phi_{x}-\phi_{y}\right\| \leq K\|x-y\|^{q-1} \text { for all } x, y \in X \backslash\{0\} .
$$

Combining (2.2), (2.3) and (2.6) shows that $f^{\prime}$ satisfies a $(q-1)$-Hölder condition.

(b) $\Rightarrow$ (c) follows from [14, Corollary 3.5.7] (see also [6, Lemma V.3.5]) and (c) $\Rightarrow$ (d) is trivial, so we prove $(\mathrm{d}) \Rightarrow$ (a). Suppose $\|\cdot\|$ does not have modulus of smoothness of power type $q$. According to [6, Lemma IV.5.1] there exist $x_{n}$, $y_{n} \in X$ and $\varepsilon>0$ such that $x_{n} \neq y_{n}, \max \left\{\left\|x_{n}\right\|,\left\|y_{n}\right\|\right\} \geq \varepsilon$ and

$$
\left\|\phi_{x_{n}}-\phi_{y_{n}}\right\| \geq n\left\|x_{n}-y_{n}\right\|^{q-1} \text { for all } n \in \mathbb{N} \text {. }
$$

This implies $\left\|x_{n}-y_{n}\right\| \rightarrow 0$. Consequently, there exists $N \in \mathbb{N}$ so that

$$
\min \left\{\left\|x_{n}\right\|,\left\|y_{n}\right\|\right\} \geq \varepsilon / 2 \text { for all } n \geq N .
$$

Now put $\tilde{x}_{n}=x_{n} /\left\|x_{n}\right\|$ and $\tilde{y}_{n}=y_{n} /\left\|y_{n}\right\|$ for $n \geq N$. Then, for $n \geq N$,

$$
\left\|\tilde{x}_{n}-\tilde{y}_{n}\right\| \leq\left\|\frac{x_{n}}{\left\|x_{n}\right\|}-\frac{y_{n}}{\left\|x_{n}\right\|}\right\|+\left\|\frac{y_{n}}{\left\|x_{n}\right\|}-\frac{y_{n}}{\left\|y_{n}\right\|}\right\| \leq \frac{4}{\varepsilon}\left\|x_{n}-y_{n}\right\| .
$$

Let $\delta_{n}=\left\|x_{n}-y_{n}\right\|$ and define $u_{n}=\frac{1}{\delta_{n} \sqrt{n}} \tilde{x}_{n}$ and $v_{n}=\frac{1}{\delta_{n} \sqrt{n}} \tilde{y}_{n}$ for $n \geq N$. Then $\left\|u_{n}-v_{n}\right\| \leq \frac{4}{\varepsilon \sqrt{n}} \rightarrow 0$. However

$$
\begin{aligned}
\left\|f^{\prime}\left(u_{n}\right)-f^{\prime}\left(v_{n}\right)\right\| & =\|q\| u_{n}\left\|^{q-1} \phi_{u_{n}}-q\right\| v_{n}\left\|^{q-1} \phi_{v_{n}}\right\| \\
& =\|q\| u_{n}\left\|^{q-1} \phi_{x_{n}}-q\right\| v_{n}\left\|^{q-1} \phi_{y_{n}}\right\| \\
& =\frac{q}{\delta_{n}^{q-1} n^{\frac{q-1}{2}}}\left\|\phi_{x_{n}}-\phi_{y_{n}}\right\| \\
& \geq \frac{q}{\delta_{n}^{q-1} n^{\frac{q-1}{2}}}\left(n \delta_{n}^{q-1}\right)=q n^{\frac{3-q}{2}} \rightarrow \infty .
\end{aligned}
$$

Consequently, $f^{\prime}$ is not uniformly continuous, and so [14, Theorem 3.5.6] (see also [6. Lemma V.3.5]) shows that $f=\|\cdot\|^{q}$ is not a uniformly smooth function.

The results in [1] enable us to derive the dual version of Theorem 2.2 for uniformly convex functions.

Theorem 2.3. Let $(X,\|\cdot\|)$ be a Banach space, and let $2 \leq p<\infty$. Then the following are equivalent.

(a) The norm $\|\cdot\|$ on $X$ has modulus of convexity of power type $p$.

(b) The function $f=\|\cdot\|^{p}$ has modulus of convexity of power type $p$.

(c) The function $f=\|\cdot\|^{p}$ is uniformly convex. 
Proof. (a) $\Rightarrow($ b): Let us assume that $\|\cdot\|$ has modulus of convexity of power type $p$. Then the modulus of smoothness of the dual norm on $X^{*}$, which we denote in this proof as $\|\cdot\|_{*}$, is of power type $q$, where $\frac{1}{p}+\frac{1}{q}=1$; see [6. Proposition IV.1.12]. By Theorem 2.2 the function $g=\frac{1}{q}\|\cdot\|_{*}^{q}$ has modulus of smoothness of power type $q$. The Fenchel conjugate of $g$ is $g^{*}=\frac{1}{p}\|\cdot\|^{p}$; see [1, 14]. Now $g^{*}$-and hence $\|\cdot\|^{p}$ — has a modulus of convexity of power type $p$ according to [1] (see also [14, Corollary 3.5.11]).

(b) $\Rightarrow$ (c) is trivial, so we prove (c) $\Rightarrow$ (a). Assume that $f=\|\cdot\|^{p}$ is a uniformly convex function. Then [1] shows that the Fenchel conjugate $f^{*}$ (and hence $\|\cdot\|_{*}^{q}$ ) is a uniformly smooth function. According to Theorem [2.2, $\|\cdot\|_{*}$ has modulus of smoothness of power type $q$; therefore $\|\cdot\|$ has modulus of convexity of power type $p$; see [6, Proposition IV.1.12].

We conclude this section by showing that the spaces with nontrivial uniformly convex functions are those that admit equivalent uniformly convex norms.

Theorem 2.4. Let $(X,\|\cdot\|)$ be a Banach space. Then the following are equivalent. (a) There exists a continuous uniformly convex function $f$ defined on $B_{X}$.

(b) $X$ admits an equivalent uniformly convex norm.

(c) There exist $p \geq 2$ and an equivalent norm $|\cdot|$ on $X$ so that the function $f=|\cdot|^{p}$ is uniformly convex.

Proof. (a) $\Rightarrow$ (b): By replacing $f$ with the function $x \mapsto \frac{f(x)+f(-x)}{2}$ we may and do assume that $f$ is symmetric, and a suitable shift guarantees that $f(0)=0$. It then follows that $f(x) \geq 0$ for all $x \in X$. Because $f$ is convex and continuous on $B_{X}$, we can fix $\varepsilon \in(0,1)$ such that $f$ is Lipschitz on $\varepsilon B_{X}$ (see [14, Corollary 2.2.13]). Let us consider the norm $\|\cdot\|$ whose unit ball is $B=\left\{x \in B_{X}: \quad f(x) \leq \delta_{f}(\varepsilon)\right\}$. The continuity of $f$ at 0 implies $0 \in \operatorname{int} B$. Moreover, $B \subset \varepsilon B_{X}$. This follows because

$$
f(x) \geq 2\left[\frac{1}{2} f(x)+\frac{1}{2} f(0)-\frac{1}{2} f\left(\frac{x}{2}\right)\right] \geq 2 \delta_{f}(\varepsilon)>\delta_{f}(\varepsilon) \text { if }\|x\|>\varepsilon .
$$

Thus, $\|\cdot\|$ is an equivalent norm on $X$.

Consider $x_{n}, y_{n} \in B_{X}$ such that $\left\|x_{n}\right\|=\left\|y_{n}\right\|=1$ and $\left\|x_{n}+y_{n}\right\| \rightarrow 2$. Because $f$ is Lipschitz on $B$, we have that $f\left(\frac{x_{n}+y_{n}}{2}\right) \rightarrow \delta_{f}(\varepsilon)$. Consequently $\frac{1}{2} f\left(x_{n}\right)+\frac{1}{2} f\left(y_{n}\right)-f\left(\frac{x_{n}+y_{n}}{2}\right) \rightarrow 0$. Thus, the uniform convexity of $f$ ensures that $\left\|x_{n}-y_{n}\right\| \rightarrow 0$ and hence $\left\|x_{n}-y_{n}\right\| \rightarrow 0$.

(b) $\Rightarrow$ (c): From (b) it follows that $X$ is superreflexive (see for instance 6, Theorem IV.4.1]). Then, there exist $p \geq 2$ and an equivalent norm $|\cdot|$ whose modulus of convexity is of power type $p$ (see [6, Theorem IV.4.8]). Consequently, Theorem 2.3 ensures that the function $f=|\cdot|^{p}$ is uniformly convex.

(c) $\Rightarrow$ (a): This is trivial.

\section{Growth RATES OF UNIFORMLY CONVEX FUNCTIONS AND RENORMING}

In this section we will construct a uniformly convex norm whose modulus of convexity is related to the growth rate of a given uniformly convex function on the Banach space. We begin with some preliminary results.

Lemma 3.1. Let $\|\cdot\|$ be a norm on a Banach space $X$. Consider $x, y \in X$ such that $\|x\|=\|y\|$, and $\|x-y\| \geq \delta$ where $0<\delta \leq 2\|x\|$. Then $\inf _{t \geq 0}\|x-t y\| \geq \delta / 2$. 
Proof. Assume that $\left\|x-t_{0} y\right\|<\delta / 2$ for some $t_{0} \geq 0$. Then $\left|1-t_{0}\right|\|y\|<\delta / 2$ and so

$$
\|x-y\| \leq\left\|x-t_{0} y\right\|+\left|1-t_{0}\right|\|y\|<\delta,
$$

which is a contradiction.

The next lemma will be used later to estimate the modulus of convexity of a norm constructed by using level sets of a symmetric uniformly convex function.

Lemma 3.2. Let $N \in \mathbb{N}$ and consider norms $\|\cdot\|_{N},\|\cdot\|_{N+1}, \ldots$ on $(X,\|\cdot\|)$ satisfying

$$
\frac{1}{2^{n+1}}\|\cdot\| \leq\|\cdot\|_{n} \leq \frac{1}{2^{n}}\|\cdot\| \text { for } n \geq N
$$

For each $n \geq N$, suppose there exists $d_{n}>0$ so that

$$
\left\|\frac{x+y}{2}\right\|_{n} \leq 1-d_{n}, \quad \text { whenever } x, y \in X,\|x\|_{n}=\|y\|_{n}=1 \text { and }\|x-y\| \geq 1 .
$$

Then there exist an equivalent norm $|\cdot|$ on $X$ and $M \in \mathbb{N}$ so that

$$
\delta_{|\cdot|}(t) \geq \frac{d_{n}}{n^{2}} \text { whenever } 2^{M+1-n} \leq t \leq 2 \text { and } n \geq M
$$

Proof. Put $M=\max \{4, N\}$, and define $|\cdot|$ by

$$
|\cdot|=\sum_{m=M}^{\infty} \frac{2^{m+1}}{m^{2}}\|\cdot\|_{m} .
$$

Observe that $|\cdot| \leq \sum_{m=M}^{\infty} \frac{2^{m+1}}{m^{2} 2^{m}}\|\cdot\| \leq \sum_{m=4}^{\infty} \frac{2}{m^{2}}\|\cdot\| \leq\|\cdot\| ;$ then

$$
\frac{1}{2^{M}}\|\cdot\| \leq \frac{1}{M^{2}}\|\cdot\| \leq \frac{2^{M+1}}{M^{2}}\|\cdot\|_{M} \leq|\cdot| \leq\|\cdot\| .
$$

Fix $n \geq M$ and $2^{M+1-n} \leq t \leq 2$. Consider $x, y \in X$ such that $|x|=|y|=1$ and $|x-y|=t$. Because $|x|=|y|=1$, it follows from (3.2) that

$$
1 \leq\|x\| \leq 2^{M} \quad \text { and } \quad 1 \leq\|y\| \leq 2^{M} .
$$

We assume, without loss of generality, $\|x\|_{n} \leq\|y\|_{n}$. Now let us denote $a=\|x\|_{n}^{-1}$ and $b=\|y\|_{n}^{-1}$. It follows from (3.1) and (3.3) that $2^{n-M} \leq b \leq a \leq 2^{n+1}$, which in turn implies $|a x-a y| \geq 2$.

According to Lemma 3.1, $|a x-b y| \geq 1$, and hence $\|a x-b y\| \geq 1$. Thus we can estimate

$$
\begin{aligned}
\left\|\frac{a x+a y}{2}\right\|_{n} & \leq\left\|\frac{a x+b y}{2}\right\|_{n}+\frac{1}{2}(a-b)\|y\|_{n} \\
& \leq \frac{1}{2}\|a x\|_{n}+\frac{1}{2}\|b y\|_{n}+\frac{1}{2}(a-b)\|y\|_{n}-d_{n} \\
& =\frac{a}{2}\left(\|x\|_{n}+\|y\|_{n}\right)-d_{n} .
\end{aligned}
$$

This inequality implies

$$
\left\|\frac{x+y}{2}\right\|_{n} \leq \frac{1}{2}\|x\|_{n}+\frac{1}{2}\|y\|_{n}-\frac{d_{n}}{a} .
$$


Thus, using (3.4) and the triangle inequality for $\|\cdot\|_{j}$ when $j \neq n$, and then that $a \leq 2^{n+1}$, we obtain

$$
\left|\frac{x+y}{2}\right| \leq \sum_{j=M}^{\infty} \frac{2^{j+1}}{2 j^{2}}\|x\|_{j}+\sum_{j=M}^{\infty} \frac{2^{j+1}}{2 j^{2}}\|y\|_{j}-\frac{2^{n+1} d_{n}}{n^{2} a} \leq 1-\frac{d_{n}}{n^{2}},
$$

which finishes the proof.

Lemma 3.3. Let $(X,\|\cdot\|)$ be a Banach space and let $f: X \rightarrow(-\infty,+\infty)$ be a symmetric, continuous, and uniformly convex function, with $f(0)<0$. Then $f(x)>0$ whenever $x \in X$ and $\|x\|>\max \left\{-f(0) /\left(2 \delta_{f}(1)\right), 1\right\}$.

Proof. The symmetry of $f$ implies that $f \geq f(0)$. Also, the continuity and uniform convexity of $f$ guarantees that $f$ is also convex. Now, take any $x \in X$ as in the conclusion of the lemma. Then

$$
\begin{aligned}
\delta_{f}(1) & \leq \frac{1}{2} f(0)+\frac{1}{2} f\left(\frac{x}{\|x\|}\right)-f\left(\frac{x}{2\|x\|}\right) \leq \frac{1}{2} f(0)+\frac{1}{2} f\left(\frac{x}{\|x\|}\right)-f(0) \\
& \leq-\frac{1}{2} f(0)+\frac{1}{2}\left[\left(1-\frac{1}{\|x\|}\right) f(0)+\frac{1}{\|x\|} f(x)\right]=-\frac{1}{2\|x\|} f(0)+\frac{1}{\|x\|} f(x),
\end{aligned}
$$

and hence, $f(x) \geq\|x\| \delta_{f}(1)+\frac{1}{2} f(0)>0$.

Proposition 3.4. Let $(X,\|\cdot\|)$ be a Banach space and let $F:[0,+\infty) \rightarrow[0,+\infty)$ be a continuous convex function satisfying $F(0)=0$. Suppose $f: X \rightarrow \mathbb{R}$ is a continuous symmetric uniformly convex function satisfying $f(x) \leq F(\|x\|)$ for all $x \in X$. Then there exists an equivalent norm $|\cdot|$ on $X$ such that given any $\gamma>0$, there are constants $\alpha>0$ and $\beta>0$ so that

$$
\delta_{|\cdot|}(t) \geq \frac{\alpha}{F\left(\beta t^{-1}\right)} t^{\gamma} \quad \text { for } 0<t \leq 2 .
$$

Proof. Because $F(0)=0$, the convexity of $F$ ensures that $F(\lambda t) \leq \lambda F(t)$ for $0 \leq \lambda \leq 1$; hence $F$ is nondecreasing on $[0,+\infty)$ because it is nonnegative there. Consequently, $F \circ\|\cdot\|$ is a convex function. According to Lemma 3.3. there exists $N \in \mathbb{N}$ so that $f(x) \geq 0$ if $\|x\| \geq N$. Now replace $f$ with $[f+F \circ\|\cdot\|] / 2$. Obviously, this new $f$ will still be uniformly convex. We have

$$
F\left(\frac{\|x\|}{2}\right) \leq \frac{1}{2} F(\|x\|) \leq f(x) \leq F(\|x\|) \text { whenever }\|x\| \geq N .
$$

For $n \geq N$, let $\|\cdot\|_{n}$ be the norm whose unit ball is $B_{n}=\left\{x \in X: \quad f(x) \leq F\left(2^{n}\right)\right\}$. It follows from (3.5) that if $\|x\|_{n}=1$, then $2^{n} \leq\|x\| \leq 2^{n+1}$. Consequently,

Let

$$
\frac{1}{2^{n+1}}\|\cdot\| \leq\|\cdot\|_{n} \leq \frac{1}{2^{n}}\|\cdot\| \text {. }
$$

$$
M_{n}=\sup \left\{f_{+}^{\prime}(u, v): \quad u, v \in X, \quad\|u\|_{n}=\|v\|_{n}=1\right\} .
$$

If $u, v$ are such, we have $\|u\| \geq 2^{n}\|u\|_{n}=2^{n} \geq 2^{N}>N$ and hence

$$
\begin{aligned}
f_{+}^{\prime}(u, v) & \leq f(u+v)-f(u) \leq f(u+v) \leq F(\|u+v\|) \\
& \leq F\left(2^{n+1}\|u+v\|_{n}\right) \leq F\left(2^{n+2}\right) .
\end{aligned}
$$

Therefore, $M_{n} \leq F\left(2^{n+2}\right)$.

Now consider $x, y \in X$ such that $\|x\|_{n}=\|y\|_{n}=1$ and $\|x-y\| \geq 1$. We will verify the hypothesis of Lemma 3.2. Put $z=\frac{x+y}{2}$ and $z^{\prime}=\frac{x+y}{\|x+y\|_{n}}$. We observe 
that $f(x)=f(y)=f\left(z^{\prime}\right)=F\left(2^{n}\right)$. Then, using the definition of $\delta_{f}(1)$ and the convexity of $f$, we have

$$
\begin{aligned}
\delta_{f}(1) & \leq \frac{1}{2} f(x)+\frac{1}{2} f(y)-f\left(\frac{x+y}{2}\right)=f\left(z^{\prime}\right)-f(z) \\
& \leq f_{+}^{\prime}\left(z^{\prime}, z^{\prime}-z\right) \leq M_{n}\left\|z^{\prime}-z\right\|_{n},
\end{aligned}
$$

and hence $\left\|z^{\prime}-z\right\|_{n} \geq \delta_{f}(1) / M_{n}$. Therefore

$$
\left\|\frac{x+y}{2}\right\|_{n}=1-\left\|z-z^{\prime}\right\|_{n} \leq 1-\frac{\delta_{f}(1)}{M_{n}} \leq 1-\frac{\delta_{f}(1)}{F\left(2^{n+2}\right)} .
$$

Applying Lemma 3.2, we find an equivalent norm $|\cdot|$ and $M \geq N$ such that

$$
\delta_{|\cdot|}(t) \geq \frac{\delta_{f}(1)}{F\left(2^{n+2}\right)} \cdot \frac{1}{n^{2}}, \quad \text { whenever } n \geq M \text { and } 2^{M+1-n} \leq t \leq 2 .
$$

Fix any $\gamma>0$. Find $n_{0}>M$ so large that $n^{-2} \geq\left(2^{-n}\right)^{\gamma}$ for all $n \geq n_{0}$. Then

$$
\delta_{|\cdot|}(t) \geq \frac{\delta_{f}(1)}{F\left(2^{n+2}\right)} \cdot\left(\frac{1}{2^{n}}\right)^{\gamma} \text { whenever } n \geq n_{0} \text { and } 2^{M+1-n} \leq t \leq 2 .
$$

Let $\alpha=\delta_{f}(1)\left(\frac{1}{2^{n_{0}+1}}\right)^{\gamma}$ and $\beta=2^{n_{0}+3}$. The previous inequality, along with the fact that $F$ is nondecreasing, ensures that for $2^{M+1-n_{0}} \leq t \leq 2$ we have

$$
\delta_{|\cdot|}(t) \geq \frac{\delta_{f}(1)}{F\left(2^{n_{0}+2}\right)}\left(\frac{1}{2^{n_{0}}}\right)^{\gamma} \geq \frac{\delta_{f}(1)}{F\left(2^{n_{0}+3} t^{-1}\right)}\left(\frac{t}{2^{n_{0}+1}}\right)^{\gamma}=\frac{\alpha t^{\gamma}}{F\left(\beta t^{-1}\right)},
$$

and for $2^{M+1-n} \leq t \leq 2^{M+2-n}$ where $n \geq n_{0}+1$, we have

$$
\delta_{|\cdot|}(t) \geq \frac{\delta_{f}(1)}{F\left(2^{n+2}\right)}\left(\frac{1}{2^{n}}\right)^{\gamma} \geq \frac{\delta_{f}(1)}{F\left(2^{M+4} t^{-1}\right)}\left(\frac{t}{2^{M+2}}\right)^{\gamma} \geq \frac{\alpha t^{\gamma}}{F\left(\beta t^{-1}\right)} .
$$

Altogether, $\delta_{|\cdot|}(t) \geq \frac{\alpha}{F\left(\beta t^{-1}\right)} t^{\gamma}$ for all $0<t \leq 2$, as desired.

Theorem 3.5. Let $(X,\|\cdot\|)$ be a Banach space, and let $p \geq 2$. Suppose $f: X \rightarrow \mathbb{R}$ is a continuous uniformly convex function such that $f(x) \leq\|x\|^{p}$ for all $x \in X$. Then for any $r>p, X$ admits an equivalent norm with modulus of convexity of power type $r$.

Proof. Apply Proposition 3.4 with $F(t)=t^{p}$.

Remark 3.6. It should be noted that $p<2$ cannot be in Theorem 3.5 since by [14, Proposition 3.5.8], $\liminf _{\|x\| \rightarrow \infty} f(x) /\|x\|^{2}>0$.

In the case $p=2$ we will prove the following sharp result.

Theorem 3.7. Let $(X,\|\cdot\|)$ be a Banach space. Then there is a continuous uniformly convex funtion $f: X \rightarrow \mathbb{R}$ satisfying $f \leq\|\cdot\|^{2}$ if and only if $X$ admits an equivalent norm with modulus of convexity of power type 2 . 
Before proving this theorem, we will present a preliminary lemma, and we also refer the reader to [7] for some related information about this case.

Lemma 3.8. Let $(X,\|\cdot\|)$ be a Banach space and $N \in \mathbb{N}$. Suppose that $|\cdot|_{N}$, $|\cdot|_{N+1}, \ldots$ are norms on $X$ such that

$$
K\|\cdot\| \leq|\cdot|_{n} \leq\|\cdot\|,
$$

for some $K>0$ and all $n \geq N$. Then there exists an equivalent norm $|\cdot|$ such that

$$
\delta_{|\cdot|}(t) \geq \liminf _{n \rightarrow \infty} \delta_{|\cdot|_{n}}(t), \quad \text { for } \quad 0<t<2 .
$$

Proof. Let us consider a free (i.e., nonprincipal) ultrafilter $\mathcal{U}$ on $\mathbb{N}$. Then $\lim _{\mathcal{U}}|x|_{n}$ exists for each $x \in X$, where $\lim _{\mathcal{U}}|x|_{n}=L$ means that for each $\varepsilon>0$, there exists $A \in \mathcal{U}$ such that $\left.|| x\right|_{n}-L \mid<\varepsilon$ for all $n \in A$. Now define $|\cdot|: X \rightarrow[0,+\infty)$ by

$$
|x|=\lim _{\mathcal{U}}|x|_{n}, \quad \text { for all } \quad x \in X .
$$

The definition of $|\cdot|$ together with (3.9) ensures that $|\cdot|$ is an equivalent norm on $X$.

We will proceed by reductio ad absurdum. Assume there is $t \in(0,2)$ such that $\delta_{|\cdot|}(t)<\lim \inf \delta_{|\cdot|_{n}}(t)$. Since $\delta_{|\cdot|}$ is continuous - see [9] - there exists $t^{\prime} \in(t, 2)$ such that $\delta_{|\cdot|}\left(t^{\prime}\right)<\liminf \delta_{|\cdot|_{n}}(t)$. Then, there exist $x, y \in X$ and a constant $a>0$ such that $|x|=|y|=1,|x-y| \geq t^{\prime}$ and $1-|(x+y) / 2|<a<\liminf \delta_{|\cdot|_{n}}(t)$. For $n \in \mathbb{N}$, let $x_{n}=x /|x|_{n}$ and $y_{n}=y /|y|_{n}$. By the definition of $|\cdot|$, there exists $A \in \mathcal{U}$ such that $\left|x_{m}-y_{m}\right|_{m}>t$ and $1-\left|\left(x_{m}+y_{m}\right) / 2\right|_{m}<a$ for all $m \in A$. Therefore $\delta_{|\cdot|_{m}}(t)<a<\liminf \delta_{|\cdot|_{n}}(t)$ for all $m \in A$, which yields a contradiction, since $\mathcal{U}$ is free and then $A$ is infinite.

Proof (of Theorem 3.7). Suppose $(X,\|\cdot\|$ ) admits an equivalent norm $|\cdot|$ that has modulus of convexity of power type 2 . We may and do assume that $|\cdot|$ has modulus of convexity of power type 2 and satisfies $|\cdot| \leq\|\cdot\|$. According to Theorem 2.3. $f=|\cdot|^{2}$ is uniformly convex as desired.

Conversely, suppose $f: X \rightarrow \mathbb{R}$ is a uniformly convex function such that $f \leq$ $\|\cdot\|^{2}$. Proceeding as in Theorem 3.4 when $F(t)=t^{2}$ we obtain norms $\left\{\|\cdot\|_{n}\right\}_{n \geq N}$ satisfying $\frac{1}{2^{n+1}}\|\cdot\| \leq\|\cdot\|_{n} \leq \frac{1}{2^{n}}\|\cdot\|$, and then (3.8) becomes

$$
\left\|\frac{x+y}{2}\right\|_{n} \leq 1-\frac{\delta_{f}(1)}{16}\left(\frac{1}{2^{n}}\right)^{2} \text {, whenever }\|x\|_{n}=\|y\|_{n}=1 \text { and }\|x-y\|_{n} \geq \frac{1}{2^{n}} .
$$

The previous inequality implies

$$
\delta_{\|\cdot\|_{n}}\left(2^{-n}\right) \geq \frac{\delta_{f}(1)}{16}\left(2^{-n}\right)^{2} .
$$

According to [8, Corollary 11] there is a universal constant $L>0$ such that

$$
\frac{\delta_{\|\cdot\|_{n}}\left(2^{-n}\right)}{\left(2^{-n}\right)^{2}} \leq 4 L \frac{\delta_{\|\cdot\|_{n}}(\eta)}{\eta^{2}} \text { for } 2^{-n} \leq \eta \leq 2 .
$$

Let $R=\frac{\delta_{f}(1)}{64 L}$; then the previous two inequalities imply

$$
\delta_{\|\cdot\|_{n}}(t) \geq R t^{2} \text { for } 2^{-n} \leq t \leq 2 .
$$


For each $n \geq N$, let us consider the new norm $|\cdot|_{n}=2^{n}\|\cdot\|_{n}$. These new norms satisfy $\frac{1}{2}\|\cdot\| \leq|\cdot|_{n} \leq\|\cdot\|$ and $\delta_{|\cdot|_{n}}(\cdot)=\delta_{\|\cdot\|_{n}}(\cdot)$. Let $|\cdot|$ be the norm found for our $|\cdot|_{N},|\cdot|_{N+1}, \ldots$ in Lemma 3.8. Then (3.10) yields that

$$
\delta_{|\cdot|}(t) \geq \liminf _{n \rightarrow \infty} \delta_{|\cdot|_{n}}(t)=\liminf _{n \rightarrow \infty} \delta_{\|\cdot\|_{n}}(t) \geq R t^{2} \text { for } 0<t \leq 2,
$$

which finishes the proof.

It does not appear as though the proof of Theorem 3.7 can be adapted to work in the case $p>2$. This is because the analogue of Figiel's result ([8, Corollary 11]) is not valid when $p>2$. Indeed, if $\delta_{\|\cdot\|}$ has modulus of power type less than $p$ for some norm $\|\cdot\|$, then $\lim \delta_{\|\cdot\|}(\varepsilon) / \varepsilon^{p} \rightarrow \infty$ as $\varepsilon \rightarrow 0$; hence a Figiel constant does not exist. However we have not checked whether such a constant exists for the family of norms $\|\cdot\|_{n}$ used in Proposition 3.4.

\section{ACKNOWLEDGMENTS}

We thank Professor C. Zălinescu and an anonymous referee for several helpful comments which led to improvements in both the accuracy and presentation of this paper.

\section{REFERENCES}

1. D. Azé and J-P. Penot, Uniformly convex and uniformly smooth convex functions, Ann. Fac. Sci. Toulouse Math. (6) 4 (1995), no. 4, 705-730. MR1623472 (99c:49015)

2. H. H. Bauschke, J. M. Borwein, and P. L. Combettes, Essential smoothness, essential strict convexity, and Legendre functions in Banach spaces, Communications in Contemporary Mathematics 3 (2001), 615-647. MR1869107 (2002k:49040)

3. D. Butnariu, A. N. Iusem, and E. Resmerita, Total convexity for powers of the norm in uniformly convex Banach spaces, J. Convex Anal. 7 (2000), no. 2, 319-334. MR 1811683 (2001m:46013)

4. D. Butnariu, A. N. Iusem, and C. Zălinescu, On uniform convexity, total convexity and convergence of the proximal point and outer Bregman projection algorithms in Banach spaces, J. Convex Anal. 10 (2003), no. 1, 35-61. MR.1999901(2004e:90161)

5. D. Butnariu and E. Resmerita, Bregman distances, totally convex functions, and a method for solving operator equations in Banach spaces, Abstr. Appl. Anal. (2006), Art. ID 84919, 39 pp. MR2211675 (2006k:47142)

6. R. Deville, G. Godefroy, and V. Zizler, Smoothness and Renormings in Banach Spaces, Pitman Monographs and Surveys in Pure and Applied Mathematics, vol. 64, Longman Scientific \& Technical, Harlow, 1993. MR 1211634 (94d:46012)

7. J. Duda, L. Veselý, and L. Zajíček, On d.c. functions and mappings, Atti Sem. Mat. Fis. Univ. Modena 51 (2003), no. 1, 111-138. MR1993883 (2004f:49030)

8. T. Figiel, On the moduli of convexity and smoothness, Studia Math. 56 (1976), no. 2, 121-155. MR0425581 (54:13535)

9. V. I. Gurariı, Differential properties of the convexity moduli of Banach spaces, Mat. Issled. 2 (1967), no. vyp. 1, 141-148. MR0211245 (35:2127)

10. E. S. Levitin and B. T. Poljak, Convergence of minimizing sequences in problems on the relative extremum, Dokl. Akad. Nauk SSSR 168 (1966), 997-1000. MR0199016 (33:7166)

11. G. Nordlander, The modulus of convexity in normed linear spaces, Ark. Mat. 4 (1960), 15-17 (1960). MR0140915 (25:4329)

12. K. R. Stromberg, An Introduction to Classical Real Analyis, Wadsworth International Mathematics Series, Wadsworth, Belmont, California, 1981. MR604364 (82c:26002) 
13. C. Zălinescu, On uniformly convex functions, J. Math. Anal. Appl. 95 (1983), no. 2, 344-374. MR716088 (85a:26018)

14. , Convex Analysis in General Vector Spaces, World Scientific Publishing Co. Inc., River Edge, NJ, 2002. MR1921556 (2003k:49003)

Faculty of Computer Science, Dalhousie University, Halifax, Nova Scotia B3H 1W5, Canada - And - School of Mathematical and Physical Sciences, University of Newcastle, Callaghan, New South Wales 2308, Australia

E-mail address: jonathan.borwein@newcastle.edu.au, jborwein@cs.dal.ca

Departamento de Matemáticas, Universidad de Murcia, 30100 Espinardo (Murcia), SPAIN

E-mail address: ajguirao@um.es

Mathematical Institute, AV ČR, Žitná 25, 11567 Praha 1, Czech Republic

E-mail address: hajek@math.cas.cz

Department of Mathematics, La Sierra University, Riverside, California 92515

E-mail address: jvanderw@lasierra.edu 